\title{
A New High-intensity Proton Irradiation Facility at the CERN PS East Area
}

\author{
Blerina Gkotse, Maurice Glaser, Pedro Lima, Emanuele Matli, Michael Moll, \\ Federico Ravotti ${ }^{1}$ \\ CERN
}

CH-1211, Geneva 23, Switzerland

E-mail: Federico.Ravotti@cern.ch

\begin{abstract}
The proton and mixed-field irradiation facilities in the CERN PS East Area (known as IRRAD1 and IRRAD2), were heavily and successfully used for irradiation of particle detectors, electronic components and materials since 1992. These facilities operated with particle bursts - protons with momentum of $24 \mathrm{GeV} / \mathrm{c}$ - delivered from the PS accelerator in "spills" of about $400 \mathrm{~ms}$ (slow extraction). With the increasing demand of irradiation experiments, these facilities suffered from a number of restrictions such as the space availability, the maximum achievable particle flux and several access constraints. In the framework of the AIDA project, an upgrade of these facilities has been realized during the CERN long shutdown (LS1). While the new proton facility (IRRAD) will continue to be mainly devoted to the radiation hardness studies for the High Energy Physics (HEP) experimental community, the new mixed-field facility (CHARM) will mainly host irradiation experiments for the validation of electronic systems used in accelerators such as the LHC. In this paper, we outline the characteristics of the new IRRAD proton facility in terms of layout, area equipment and potential for new irradiation experiments.
\end{abstract}

Technology and Instrumentation in Particle Physics 2014

2-6 June, 2014

Amsterdam, the Netherland

\footnotetext{
${ }^{1}$ speaker
} 


\section{Introduction}

The proton irradiation facility in the PS East Area $^{2}$ (known as IRRAD1), on the T7 beam line, was heavily and successfully exploited for irradiation of particle detector elements, electronic components and detector materials since 1992. The mixed-field irradiation facility (known as IRRAD2) was implemented behind the DIRAC experiment on the T8 beam line and has been operated parasitically to DIRAC since 1998 [1]. These facilities received particle bursts - protons with momentum of $24 \mathrm{GeV} / \mathrm{c}$ - delivered from the PS accelerator in "spills" of about 400ms (slow extraction). In IRRAD1, the proton beam was spread out in order to produce a uniform irradiation spot of several square centimetres, while in IRRAD2 the irradiation was performed in a small cavity with secondary particles produced by the primary proton beam impinging on a thick target made of carbon, iron and lead [2]. In the past 15 years, more than 8300 "pieces" were irradiated using this infrastructure.

Based on previous studies carried out during the years 2007-2010 [3], the need of improved irradiation facilities at CERN, was confirmed and consolidated. More specifically, in view of the high-luminosity upgrade of the LHC and its experiments, the old PS East Area (EA) facilities suffered from a number of restrictions:

1. the available space was very limited and allowed only the irradiation of relatively small and stand-alone objects;

2. the proton flux was limited on the one hand by the weakness of the shielding and, on the other hand, by the competition for proton spills with DIRAC;

3. the access to the IRRAD1 proton facility required long cool-down time and a stop of the operation in the whole EA for the full duration of the access.

In the framework of the AIDA project [4], an upgrade of the EA irradiation facilities on the T8 beam-line was proposed based on the assumption that DIRAC would have concluded its experimental program in 2012. The DIRAC experimental apparatus was decommissioned during 2013. Today, the new EA-IRRAD facility is being constructed in the former DIRAC beam line within the framework of the overall renovation project of the PS East Area [5]. The EA-IRRAD project involves several CERN groups belonging mainly to the Engineering and Physics department.

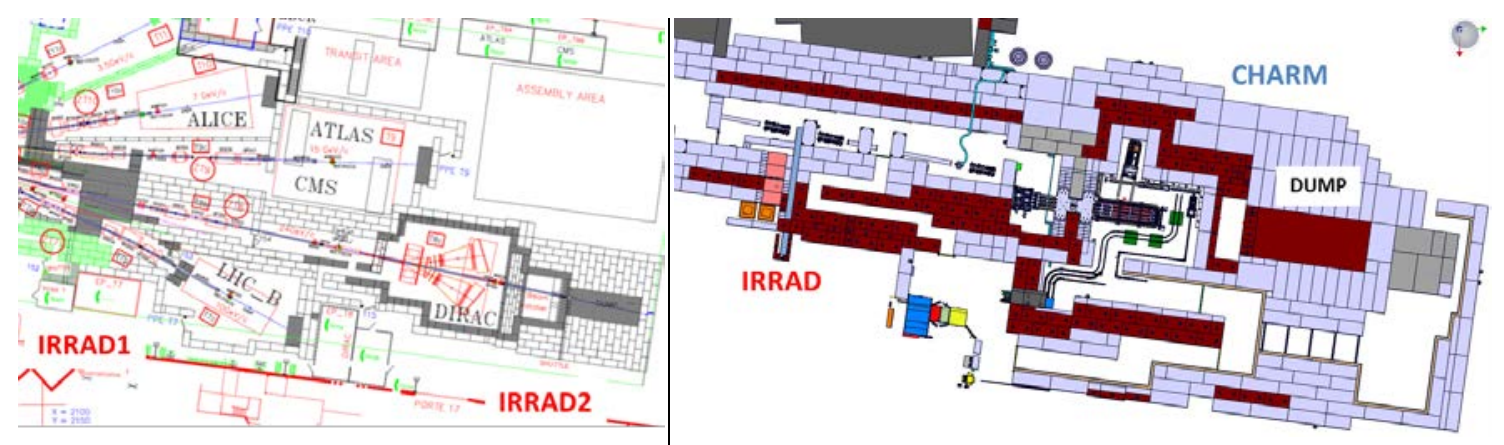

Figure 1: Layout of the PS East Area until 2012 (left). New combined EA-IRRAD facility implemented on the T8 beam-line (right): the proton area, IRRAD (upstream), is followed by the mixed-field area CHARM (downstream).

\footnotetext{
${ }^{2}$ www.cern.ch/irradiation
} 
As shown in Fig. 1 (right), the new proton irradiation facility (named IRRAD) is being constructed at the location previously occupied by the DIRAC target, while the mixed-field facility (named $\mathrm{CHARM}^{3}$ ) is constructed downstream of IRRAD, in the location where the former DIRAC detector was installed (left of Fig. 1). This solution has several advantages:

1. the access to the facility is independent of other experiments located in the PS EA;

2. the layout is optimised for exploitation as an irradiation facility, with appropriate shielding, ventilation, dedicated infrastructure and sufficient space for a proper installation and easy accessibility of the equipment;

3. the same protons are used for both IRRAD and CHARM, thus leading to a strongly improved PS cycle economy and optimal use of available protons.

In the new IRRAD facility, a particle fluence of about $1 \times 10^{16} \mathrm{p} / \mathrm{cm}^{2}$ could be reached in 5 days over a surface of $12 \times 12 \mathrm{~mm}^{2}$ (FWHM). This roughly corresponds to a factor 4-increased intensity with respect to the previous IRRAD1 facility. In the following, the key characteristics of the new IRRAD facility will be described in more detail.

\section{Layout of the IRRAD proton facility}

The IRRAD bunker is subdivided in three zones according to the nature of the samples to be irradiated. With reference to Fig. 2 (from upstream to downstream), the first zone is dedicated to the irradiation of "light" materials such as small silicon detectors, the second zone to the irradiation of intermediate materials such as electronic cards and components, while the third zone is optimized to perform irradiation experiments on "high-Z" materials such as samples of calorimeter crystals. In this last zone, it will be also possible to perform irradiations in cryogenic conditions using a dedicated setup operating with liquid Helium. Moreover, a fourth zone, in a partially shielded area, is equipped for the installation of readout electronics and/or DAQ systems that need to be close to the Devices Under Test (DUT) during irradiation.

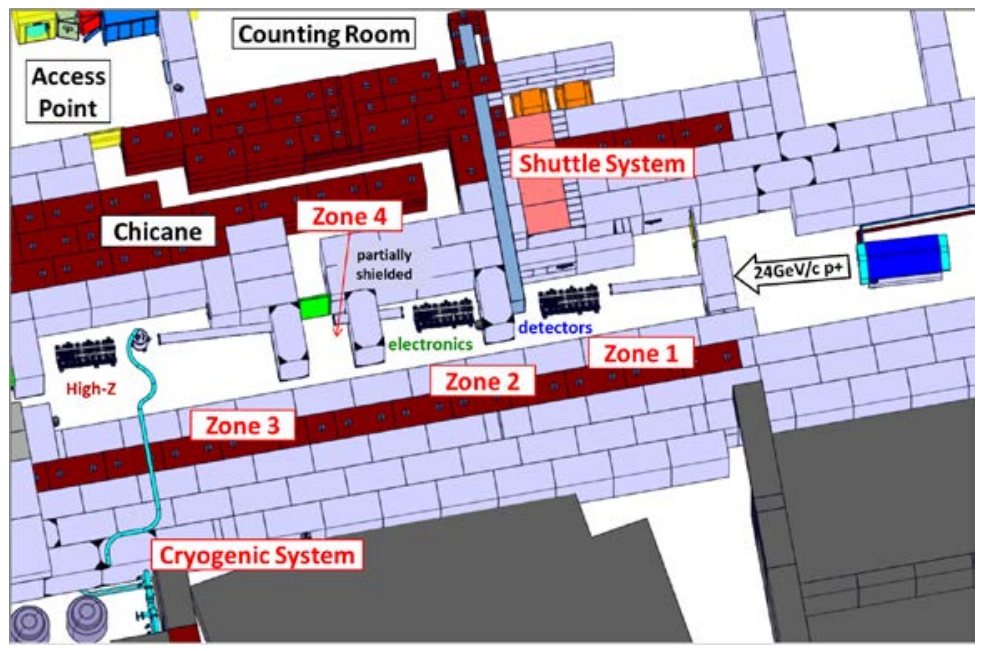

Figure 2: Detailed layout of the IRRAD proton facility.

In between each irradiation zone, an $80 \mathrm{~cm}$-thick concrete separation wall is installed in order to reduce the background during operation and to minimize the ambient dose equivalent to

${ }^{3}$ www.cern.ch/charm 
the personnel accessing the area. As visible in Fig. 2, in order to reduce further the secondary radiation produced by the interaction of the proton beam with the air, sections of vacuum beampipe are installed in the empty space between the various irradiation systems.

The DUTs are installed and positioned in the proton beam by using two different types of remote controlled holders: the irradiation tables (section 3) and the irradiation shuttle (section 4). Finally, a set of beam instruments will be installed inside the IRRAD bunker to allow a constant monitoring of the irradiation conditions (section 5).

\section{Irradiation tables}

The tables are remote-controlled stages providing the possibility to position the DUT with $\pm 0.1 \mathrm{~mm}$ precision in the transversal plane $(\mathrm{X}-\mathrm{Y})$ with respect to the beam axis. The tables also rotate over the azimuthal angle $(\theta)$ in order to achieve a precise alignment with the beam within $\pm 0.025^{\circ}$. The prototype of an irradiation table is shown on the left-hand side of Fig. 3 . The installation of the samples on the table requires the access to the proton area.

Three independent groups of tables will be installed in the three zones of the IRRAD facility bunker. A maximum of three tables per group (e.g. 9 in the whole facility) can be installed and operated. This allows the irradiation of several materials at the same time with a "clean" proton beam and minimum background induced by scattered secondary particles.

On each table, the maximum volume available for irradiation is of $200 \times 200 \times 500 \mathrm{~mm}^{3}$ while the maximum samples weight is $50 \mathrm{~kg}$. The tables can automatically move (e.g. "scan") the samples during irradiation in order to provide a uniform spot over the $200 \times 200 \mathrm{~mm}^{2}$ surface (or a smaller portion of it, depending on user request). On these tables, the test of equipment "in operation" (e.g. powered and connected to a DAQ system) is possible as well as irradiation of detector components at low temperature, down to about $-20^{\circ} \mathrm{C}$, using specially designed thermostatized irradiation boxes (see Fig. 3, left-hand side).
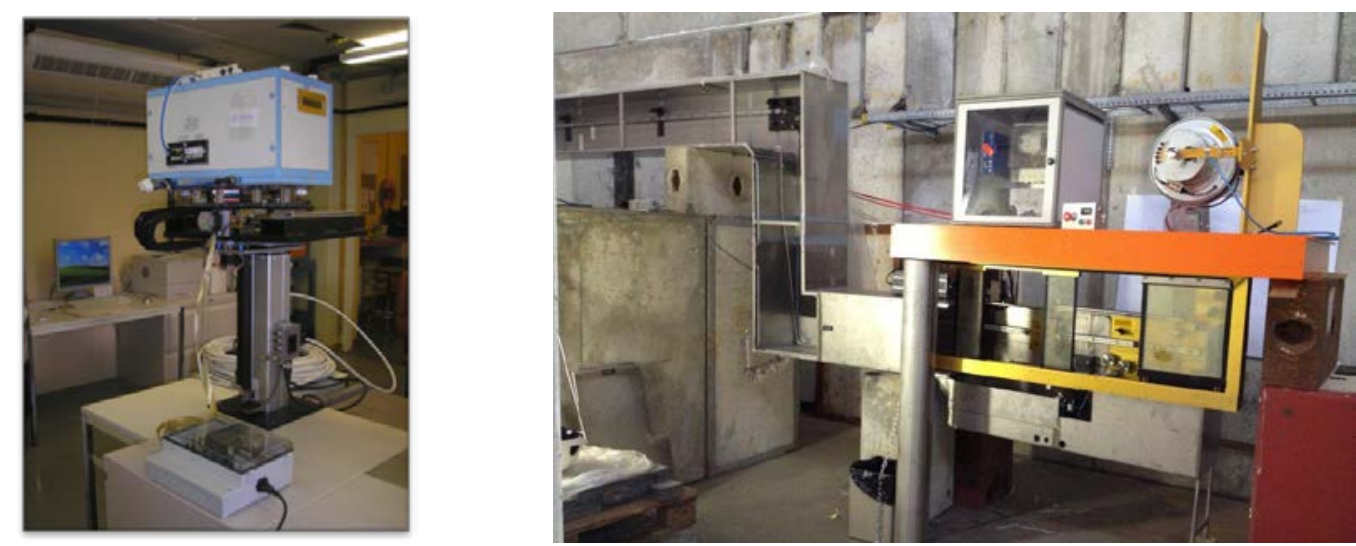

Figure 3: Prototype of a table for proton irradiation equipped with a thermostatized irradiation box (left). Loading station of the IRRAD shuttle with its motorization system (picture taken during installation).

\section{Irradiation shuttle}

The shuttle is a remote-controlled trolley travelling on a rail system and allowing the positioning of "small" objects in the beam (typically silicon detector test-samples) without the 
need of human access into the area. This system guarantees a precise $X-Y$ alignment of $\pm 0.1 \mathrm{~mm}$ with respect to the beam axis and it is manly designed for the irradiation of passive samples at room temperature. The shuttle for the new proton irradiation facility is cloned from the previous IRRAD1 and IRRAD2 shuttle systems (see Fig. 3, right-hand side). The shuttle travels across the shielding blocks for a length of about $10 \mathrm{~m}$ inside a conduit of $400 \times 400 \mathrm{~mm}^{2}$. To minimize the direct radiation streaming, the path of the conduit follows a chicane located in between Zone 1 and Zone 2 as shown in Fig. 2. On the shuttle, the maximum volume available for irradiation is of about $50 \times 50 \times 200 \mathrm{~mm}^{3}$ for a maximum weight of about $1 \mathrm{Kg}$. The standard size of the beam spot on the shuttle system $(\sigma)$ is of about $5-7 \mathrm{~mm}$ RMS but it can vary according to the different beam focusing options. In particular, focusing on the shuttle system, the spot size can be reduced further down during high-intensity irradiation periods. Two radiation monitors (Automess 6150AD6) are installed on the shuttle and are used to measure remotely the radiation levels of the irradiated samples while being removed from the irradiation area.

\section{Monitoring of the beam conditions}

The intensity of the extracted proton spills is monitored using a Secondary Emission Chamber (SEC) device provided by PS beam instrumentation. This device is installed in the upstream PS area, and its signal is made available in the counting room together with the others provided by the PS timing-distribution. The profile of the proton beam with a spill-by-spill resolution is obtained by a custom-made Beam Profile Monitor (BPM). The operation of this instrument is based on the secondary electrons emission effect. One BPM is located upstream the IRRAD area, in the same location as used for the SEC. Additional BPM devices will be distributed along the trajectory of the proton beam, to guarantee the fine tuning of the beam profile on all irradiation systems. The absolute calibration of this beam instrumentation in terms of $\mathrm{p} / \mathrm{cm}^{2}$ is obtained by means of activation measurements of thin aluminium foils.

\section{Conclusions}

This paper provides a brief overview about the key characteristics of the new IRRAD proton facility in the CERN PS East Area. The new infrastructure is currently being constructed and expected to be ready for the commissioning with the proton beam during summer 2014 . Although the EA-IRRAD infrastructure will be fully completed only during autumn 2014, first irradiation experiments in IRRAD are expected to be performed before the end of the year.

\section{References}

[1] M. Glaser, L. Durieu, C. Leroy, M. Tavlet, P. Roy and F. Lemeilleur, New irradiation zones at the CERN-PS, Nucl. Instr. and Methods A426, pp. 72-77, 1999.

[2] F. Ravotti, M. Glaser, M. Moll, Dosimetry Assessments in the Irradiation Facilities at the CERN-PS Accelerator, IEEE Trans. Nucl. Sci., 53(4), pp. 2016-2022, 2006.

[3] Working group on future irradiation facilities at CERN (http://www.cern.ch/irradiation-facilities/).

[4] AIDA Project (http://aida.web.cern.ch/aida/index.html).

[5] East Area upgrade project (http://sba.web.cern.ch/sba/BeamsAndAreas/East/East.htm). 